\title{
Correction on the articles' citations in Volumes 93/4, 93/5 and $93 / 6$
}

Received: 6 July 2021

Unfortunately, a mistake occurred in the citations of papers belonging to Volumes 93/4, 93/5 and 93/6.

Papers with full citations:

1 - Sarra Dehili, Damien Barakel, Laurent Ottaviani, Olivier Palais, Nickel and gold identification in p-type silicon through TDLS: a modeling study, Eur. Phys. J. Appl. Phys. 93, 40101 (2021) https://doi.org/10.1051/epjap/2021210015

2 - Qingwen Lan, Changpeng Chen, Tian Qin, Electronic and optical properties of Janus monolayers $\mathrm{MoXB}_{2}(\mathrm{X}=\mathrm{S}, \mathrm{Se})$ : first-principles prediction, Eur. Phys. J. Appl. Phys. 93, 40301 (2021) https://doi.org/10.1051/epjap/2021210007

3 - Abdellah Sellam, El Kebir Hlil, Rodolphe Heyd, Abdelaziz Koumina, An ab initio investigation of the electronic and magnetic properties of graphite and nickel-doped graphite, Eur. Phys. J. Appl. Phys. 93, 40401 (2021) https://doi.org/ $10.1051 / \mathrm{epjap} / 2021200217$

4 - Mengyi Wang, Rongxin Sha, Ziyang Zhang, Ailiang Zou, Yuekui Xu, Min Liu, Yibo Peng, Zhiyong Qiu, One-step synthesis of gold nanoparticles carried by boehmite, Eur. Phys. J. Appl. Phys. 93, 40402 (2021) https://doi.org/10.1051/ epjap/2021200330

5 - Leonid M. Goldenberg, Mathias Köhler, Christian Dreyer, Tohralf Krahl, Erhard Kemnitz, Optical nanocomposites containing low refractive index $\mathrm{MgF}_{2}$ nanoparticles, Eur. Phys. J. Appl. Phys. 93, 40403 (2021) https://doi.org/10.1051/ epjap/2021200298

6 - Qingyu Hou, Yuqin Guan, Zhichao Wang, Effect of different Mn doping and point vacancy ratios on the magnetic properties of ZnO, Eur. Phys. J. Appl. Phys. 93, 50101 (2021) https://doi.org/10.1051/epjap/2021210012

7 -Sanket S. Jugade, Anuj Aggarwal, Akshay K. Naik, Nanomechanical spectroscopy of ultrathin silicon nitride suspended membranes, Eur. Phys. J. Appl. Phys. 93, 50301 (2021) https://doi.org/10.1051/epjap/2021210068

8 - Yu Hao, Lan Yang, Jinze Li, Ruonan Xing, Yuzong Gu, Third-order nonlinear optical properties of CuS/reduced graphene oxide nanocomposites, Eur. Phys. J. Appl. Phys. 93, 50401 (2021) https://doi.org/10.1051/epjap/2021200278

9 - Radiyah A. Bahareth, Mai ME. Barakat, Aiyeshah Alhodaib, Saad Aldawood, Samir A. Nouh, Tailoring the optical properties of PC/ZnS nanocomposite by $\gamma$ radiation, Eur. Phys. J. Appl. Phys. 93, 50402 (2021) https://doi.org/10.1051/ epjap/2021210053

10 - Mohamed Lmouchter, Minoru Suzuki, Sustained coherent epitaxy and role of oxygen vacancies in $\operatorname{La}_{0.7} \mathrm{Sr}_{0.3} \mathrm{MnO}_{3-\delta}$ thin films grown on $\mathrm{SrTiO}_{3}$ by sputtering, Eur. Phys. J. Appl. Phys. 93, 60301 (2021) https://doi.org/10.1051/epjap/ 2021200365

11 - Leonid M. Goldenberg, Mathias Köhler, Christian Dreyer, Tohralf Krahl, Erhard Kemnitz, Erratum to: Optical nanocomposites containing low refractive index $\mathrm{MgF}_{2}$ nanoparticles, Eur. Phys. J. Appl. Phys. 93, 60401 (2021) https://doi.org/10.1051/epjap/2021210111

12 - Hammedi Khadija, Marouan Khalifa, M. Consuelo Alvarez-Galvan, Hatem Ezzaouia, Correlation between Structural and morphological properties of multilayer perovskite $\mathrm{ZnTiO}_{3}$ coated porous silicon, Eur. Phys. J. Appl. Phys. 93, 60402 (2021) https://doi.org/10.1051/epjap/2021210016

13 - Michel Feidt, Renaud Feidt, Endo-irreversible thermo-mechanical Carnot engine with new concept of entropy production action coefficient, Eur. Phys. J. Appl. Phys. 93, 60901 (2021) DOI: https://doi.org/10.1051/epjap/ 2021200390

14 - Wang Xingguo, Shu Haisheng, Zhang Lei, Vibration and acoustic insulation properties of generalized phononic crystals, Eur. Phys. J. Appl. Phys. 93, 60902 (2021) https://doi.org/10.1051/epjap/2021210036

15 - Alaa Dahshan, Horesh Kumar, Neeraj Mehta, Role of some modifiers on the thermo-mechanical properties of $\operatorname{Se}_{90} \mathrm{In}_{10}$ chalcogenide glass (ChGs), Eur. Phys. J. Appl. Phys. 93, 61101 (2021) https://doi.org/10.1051/epjap/2021210044 
Become correspondingly:

1 - Sarra Dehili, Damien Barakel, Laurent Ottaviani, Olivier Palais, Nickel and gold identification in p-type silicon through TDLS: a modeling study, Eur. Phys. J. Appl. Phys. 94, 10101 (2021) https://doi.org/10.1051/epjap/2021210015

2 - Qingwen Lan, Changpeng Chen, Tian Qin, Electronic and optical properties of Janus monolayers $\mathrm{MoXB}_{2}(\mathrm{X}=\mathrm{S}$, Se): first-principles prediction, Eur. Phys. J. Appl. Phys. 94, 10301 (2021) https://doi.org/10.1051/epjap/2021210007

3 - Abdellah Sellam, El Kebir Hlil, Rodolphe Heyd, Abdelaziz Koumina, An ab initio investigation of the electronic and magnetic properties of graphite and nickel-doped graphite, Eur. Phys. J. Appl. Phys. 94, 10401 (2021) https://doi.org/ 10.1051/epjap/2021200217

4 - Mengyi Wang, Rongxin Sha, Ziyang Zhang, Ailiang Zou, Yuekui Xu, Min Liu, Yibo Peng, Zhiyong Qiu, One-step synthesis of gold nanoparticles carried by boehmite, Eur. Phys. J. Appl. Phys. 94, 10402 (2021) https://doi.org/10.1051/ epjap/2021200330

5 - Leonid M. Goldenberg, Mathias Köhler, Christian Dreyer, Tohralf Krahl, Erhard Kemnitz, Optical nanocomposites containing low refractive index $\mathrm{MgF}_{2}$ nanoparticles, Eur. Phys. J. Appl. Phys. 94, 10403 (2021) https://doi.org/10.1051/ epjap/2021200298

6 - Qingyu Hou, Yuqin Guan, Zhichao Wang, Effect of different Mn doping and point vacancy ratios on the magnetic properties of ZnO, Eur. Phys. J. Appl. Phys. 94, 20101 (2021) https://doi.org/10.1051/epjap/2021210012

7 -Sanket S. Jugade, Anuj Aggarwal, Akshay K. Naik, Nanomechanical spectroscopy of ultrathin silicon nitride suspended membranes, Eur. Phys. J. Appl. Phys. 94, 20301 (2021) https://doi.org/10.1051/epjap/2021210068

8 - Yu Hao, Lan Yang, Jinze Li, Ruonan Xing, Yuzong Gu, Third-order nonlinear optical properties of CuS/reduced graphene oxide nanocomposites, Eur. Phys. J. Appl. Phys. 94, 20401 (2021) https://doi.org/10.1051/epjap/2021200278

9 - Radiyah A. Bahareth, Mai ME. Barakat, Aiyeshah Alhodaib, Saad Aldawood, Samir A. Nouh, Tailoring the optical properties of PC/ZnS nanocomposite by $\gamma$ radiation, Eur. Phys. J. Appl. Phys. 94, 20402 (2021) https://doi.org/ $10.1051 /$ epjap/2021210053

10 - Mohamed Lmouchter, Minoru Suzuki, Sustained coherent epitaxy and role of oxygen vacancies in $\mathrm{La}_{0.7} \mathrm{Sr}_{0.3} \mathrm{MnO}_{3-\delta}$ thin films grown on $\mathrm{SrTiO}_{3}$ by sputtering, Eur. Phys. J. Appl. Phys. 94, 30301 (2021) https://doi.org/10.1051/epjap/ 2021200365

11 - Leonid M. Goldenberg, Mathias Köhler, Christian Dreyer, Tohralf Krahl, Erhard Kemnitz, Erratum to: Optical nanocomposites containing low refractive index $\mathrm{MgF}_{2}$ nanoparticles, Eur. Phys. J. Appl. Phys. 94, 30401 (2021) https:// doi.org/10.1051/epjap/2021210111

12 - Hammedi Khadija, Marouan Khalifa, M. Consuelo Alvarez-Galvan, Hatem Ezzaouia, Correlation between Structural and morphological properties of multilayer perovskite $\mathrm{ZnTiO}_{3}$ coated porous silicon, Eur. Phys. J. Appl. Phys. 94, 30402 (2021) https://doi.org/10.1051/epjap/2021210016

13 - Michel Feidt, Renaud Feidt, Endo-irreversible thermo-mechanical Carnot engine with new concept of entropy production action coefficient, Eur. Phys. J. Appl. Phys. 94, 30901 (2021) https://doi.org/10.1051/epjap/2021200390

14 - Wang Xingguo, Shu Haisheng, Zhang Lei, Vibration and acoustic insulation properties of generalized phononic crystals, Eur. Phys. J. Appl. Phys. 94, 30902 (2021) https://doi.org/10.1051/epjap/2021210036

15 - Alaa Dahshan, Horesh Kumar, Neeraj Mehta, Role of some modifiers on the thermo-mechanical properties of $\operatorname{Se}_{90} \mathrm{In}_{10}$ chalcogenide glass (ChGs), Eur. Phys. J. Appl. Phys. 94, 31101 (2021) https://doi.org/10.1051/epjap/2021210044

The Publisher will ensure that all modifications will be correctly transmitted to the indexation bases.

The Publisher apologizes for this mistake.

Cite this article as: Correction on the articles' citations in Volumes 93/4, 93/5 and 93/6, Eur. Phys. J. Appl. Phys. 94, 30101 $(2021)$ 